\title{
Forces Applied during Transvenous Implantable Cardioverter Defibrillator Lead Removal
}

\author{
Carsten Lennerz, ${ }^{1}$ Herribert Pavaci, ${ }^{1}$ Christian Grebmer, ${ }^{1}$ \\ Gesa von Olshausen, ${ }^{2}$ Verena Semmler, ${ }^{1}$ Alessandra Buiatti, ${ }^{1}$ Tilko Reents, ${ }^{1}$ \\ Sonia Ammar, ${ }^{1}$ Isabel Deisenhofer, ${ }^{1}$ and Christof Kolb ${ }^{1}$ \\ ${ }^{1}$ Deutsches Herzzentrum München, Klinik für Herz- und Kreislauferkrankungen, Fakultät für Medizin, \\ Technische Universität München, Lazarettstraße 36, 80636 München, Germany \\ ${ }^{2}$ Klinikum rechts der Isar, 1. Medizinische Klinik, Fakultät für Medizin, Technische Universität München, Ismaninger Straße 2, \\ 81675 München, Germany
}

Correspondence should be addressed to Carsten Lennerz; lennerz@dhm.mhn.de

Received 11 December 2013; Revised 9 April 2014; Accepted 29 April 2014; Published 21 May 2014

Academic Editor: Henny C. van der Mei

Copyright ( 2014 Carsten Lennerz et al. This is an open access article distributed under the Creative Commons Attribution License, which permits unrestricted use, distribution, and reproduction in any medium, provided the original work is properly cited.

\begin{abstract}
Methods. 17 physicians, experienced in transvenous lead removal, performed a lead extraction manoeuvre of an ICD lead on a torso phantom. They were advised to stop traction only when further traction would be considered as harmful to the patient or whenbased on their experience- a change in the extraction strategy was indicated. Traction forces were recorded with a digital precision gauge. Results. Median traction forces on the endocardium were $10.9 \mathrm{~N}$ (range from $3.0 \mathrm{~N}$ to $24.7 \mathrm{~N}$ and interquartile range from 7.9 to 15.3). Forces applied to the proximal end were estimated to be $10 \%$ higher than those measured at the tip of the lead due to a friction loss.Conclusion. A traction force of around $11 \mathrm{~N}$ is typically exerted during standard transvenous extraction of ICD leads. A traction threshold for a safe procedure derived from a pool of experienced extractionists may be helpful for the development of required adequate simulator trainings.
\end{abstract}

\section{Introduction}

The number of implanted cardiac implantable electronic devices (CIED) has increased over the recent years [1]. This trend is caused by a wider range of indications $[2,3]$. Moreover, the number of leads per patient is increasing with cardiac resynchronization therapy and a higher proportion of dual versus single-chamber devices. Due to the increase in CIED and lead implantations, patient's longer life expectancy, extended indications for removal, and lead recalls, the number of lead extractions is expected to grow [3-7]. Every year more than 10,000-15,000 patients undergo lead extraction worldwide [7-9]. Major indications for lead extraction are infections, followed by lead revisions of functional or nonfunctional leads and thrombosis or venous stenosis $[4,10]$.

Open surgery strategies have been abandoned in favour of a highly successful transvenous technique (success rate $>98 \%$ with low morbidity and mortality) $[3,7,11,12]$. Lifethreatening complications (i.e., myocardial avulsion, cardiac tamponade, vascular tear, and pulmonary embolism) and death are reported in less than $1 \%$ of procedures [11]. Typically, the transvenous lead extraction (TLE) is initially attempted by simple manual traction with a stylet inserted to the lead's lumen [4]. This technique yields success rates of up to $29 \%$ [7, 12-15]. Advanced extraction techniques using locking stylets, nonpowered, or powered sheaths are conducted in case of simple manual extraction failure [11].

So far it is not known what objective force is being applied to either the lead or the heart during a manual extraction procedure. In fact, the maximally tolerated force during an extraction procedure is dependent on lead elongation, fluoroscopic, hemodynamic, and haptic feedback but may also vary among operators. 
The purpose of this study was to characterise traction forces acceptable to an experienced operator during standard lead extractions.

\section{Methods}

During the annual meeting of the German Cardiac Society in Mannheim 2013, cardiologists and cardiac surgeons were invited to participate in a simulation on lead extraction. Inclusion criterion for participation in the study was an experience level of at least 40 prior TLE procedures following the recommendation of the HRS/AHA and EHRA $[4,10]$. In order to represent various extracting centres in our study, we did not insist on the otherwise recommended extraction rate of $>20$ /year.

In order to measure the forces applied during extraction procedures a torso phantom was designed (Figure 1). A lead was inserted into the torso from a left-sided access. Within the torso, the lead followed an anatomic model of the subclavian and superior caval vein to the right atrium. The tip of the lead was then connected to a commercially available digital force gauge (FB 200, PCE Deutschland $\mathrm{GmbH}$, Meschede, Germany) in a virtual right ventricular apical position. The outermost part of the electrode tip was chosen as the anchoring point in order to simulate the active or passive lead-tissue fixation and to allow a realistic tension behaviour of the lead with elongation and lead disintegration at predetermined breaking points.

Participants were asked to perform a simple manual traction manoeuvre on our torso. They were supplied with an ICD electrode (Durata 7121, 7121Q, 7122, 7170Q, and 7171-all from St. Jude Medical, Sylmar, CA, USA) and the corresponding stylet. The probands were then instructed to extract the lead as in real life. They were advised to stop only when further traction would be considered harmful to the patient or when-based on their experience-a change in the extraction strategy was indicated. During the extraction manoeuvre, the traction pattern over time and the maximal traction force were recorded. The precision force gauge used in this experiment provides a resolution of $0.05 \mathrm{~N}$ within $0-$ $200 \mathrm{~N}$ and allows real time traction recording at a rate of $40 / \mathrm{sec}$. After every extraction procedure the lead elongation was quantified and the lead was replaced by a new one for the next study participant. There was no technical limitation on forces during the extraction procedures, and forces were only limited by the lead design with its elastic properties of the insulation material and its tear strength.

Our experimental setup allows the measurement of forces acting directly at the lead tip, displayed as well as stored by the force gauge. The forces effective at the proximal part of the lead were determined indirectly. For this our model was calibrated with predefined tractions using the gravitation force of standard weights $(50 \mathrm{~g}, 100 \mathrm{~g}, 200 \mathrm{~g}, 500 \mathrm{~g}, 1000 \mathrm{~g}$, and $2000 \mathrm{~g})$. The ratio between the expected force caused by the defined weights and the measured force at the lead tip defines the system-immanent traction loss. Thus understanding the traction loss in our model and knowing the effective force at the distal end of the lead, the force exerted at the proximal end can be calculated for each extraction manoeuvre.

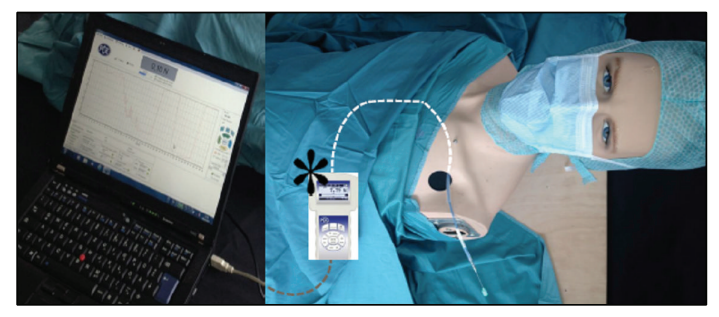

Figure 1: Torso phantom for simulation of a transvenous lead extraction procedure; white dashed line illustrates the course of the lead within the thorax. ${ }^{*}$ Digital force gauge.

In addition, background and experience level in lead extraction of each participant were assessed by self-report. The study was registered at "ClinicalTrials.gov" under NCT01847625.

2.1. Statistics. Data are presented as frequencies and percentages, and distribution of forces applied is displayed as median, minimum, and maximum value as well as interquartile range.

\section{Results}

In total, 20 probands took part in our simulator study. At the end, the data of 17 participants was evaluated since three did not meet the inclusion criteria of being well experienced in lead extraction. Most of the 17 volunteers (16 male, 1 female; 12 cardiologists, 5 cardiac surgeons) were between 40 and 50 years old (9/17) or at an age above 50 years (7/17). Eight of the probands complied with the HRS/AHA/EHRA requirements of an annual extraction rate of $>20$ leads. Furthermore, ten participants had accumulated a total extraction volume of $>100$, among them four with an extraction volume of $>400$.

The median traction force on the lead tip, which corresponds to the force applied to the endocardium, was 10.9 N (minimum $3.0 \mathrm{~N}$, maximum $24.7 \mathrm{~N}$, and interquartile range 7.9 to $15.3 \mathrm{~N}$; see Figure 2). Calibration measurements revealed that our system was afflicted with a nearly constant friction loss of $10 \%$ of the gravitation forces caused by weights between $50 \mathrm{~g}$ and $2000 \mathrm{~g}$. Thus, the force applied to the proximal end of the lead equals the measured force at the lead tip plus the friction loss in the anatomic model and therefore is $10 \%$ higher than the measured values at the tip of the lead.

Although a considerable lead elongation could be perceived by the participants during the extraction manoeuvre, none of the leads showed an elongation after the extraction manoeuvre due to the elastic properties of the leads and its reset force.

Amongst the participants, we could identify three extraction patterns. Most of the probands (12/17) gradually increased the traction force up to the individually accepted maximum and then completely released all traction and started a second attempt (Figure 3 ). In contrast, 4/17 volunteers started in the same way but then undulated the traction to their maximally accepted force over a longer period of time. One proband aimed to reach the maximum traction force in the shortest possible time. Figure 4 shows 




Figure 2: Traction force on the endocardium under a simulated extraction procedure.

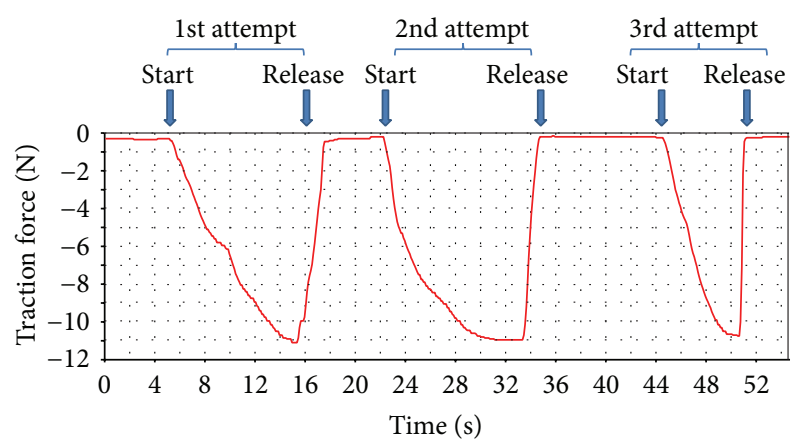

FIgURE 3: Typical extraction pattern with a moderate increase of traction up to the maximum, followed by a complete release and a new extraction attempt; the maximum force $[\mathrm{N}]$ and force increase $[\mathrm{N} / \mathrm{s}]$ for each of the extraction attempts are constant and reproducible in this highly experienced extractionist.

the distribution of traction increase over time amongst our volunteers.

\section{Discussion}

With the increasing need for lead removal TLE has become a demanded and sophisticated task $[7,11,12,16]$. Despite of the increased extraction practice, little is known on the actual forces applied during TLE. The only available data on traction forces date back to the 1980s and refer to the-nowadays obsolete-continuous traction method at the bedside $[17,18]$.

The presented investigation is the first analysis on traction forces applied to the endocardium and the lead with a contemporary TLE technique. Although there is interoperator variability in the forces exerted, typically around $11 \mathrm{~N}$ are applied and considered safe in manual traction procedures. Interestingly, the currently applied traction forces are in the same range as those used during the continuous traction era when weights of up to $3 \mathrm{lbs}(\sim 12 \mathrm{~N})$ over a maximum of 7 days were recommended.

Furthermore, the traction forces determined in our study are consistent with the traction forces required in current EN standards. According to the EN standard 45502-2-1, a lead has to resist a traction force of at least $5 \mathrm{~N}$ over at least 1 minute in order to obtain market approval.

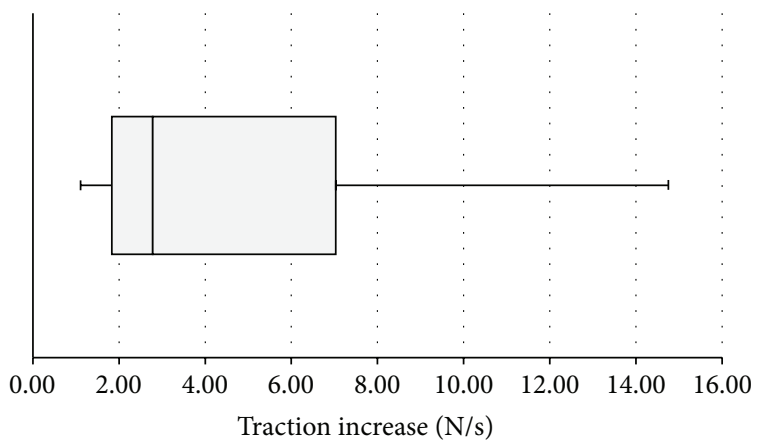

FIgURE 4: Traction increase [N/s] at the lead tip during simulated extraction procedure. One extreme value $(22.4 \mathrm{~N} / \mathrm{s})$ represents a statistical outlier and was not included in the graph.

As discussed in the HRS/AHA consensus paper and as confirmed by "real" world studies only very few extractionists meet the requirements on minimum extraction training and volume to consistently deliver safe and effective care [4, 6,10]. A survey in the United Kingdom showed that 56\% of extracting physicians perform less than 20 procedures per year [19]. Acknowledging that the requirements on competency are very difficult to achieve, the Swiss guidelines reduced the minimum requirement to 15 extractions a year [20]. In light of a discrepancy between requirements and general practice, the cardiac societies point out the need for adequate simulator training to safely practice the extraction scenario $[4,10]$. In general, studies have demonstrated an accelerated learning curve and a reduction in complications with simulator trainings. To the authors' knowledge such a simulator or training program does not yet exist. Our study may be of particular relevance for establishing such simulator training by providing the maximum threshold of traction force upcoming extractionists should be trained to. The data acquired may be of particular value since they were derived from a rare pool of TLE experts, otherwise difficult to access.

Although many extractionists start with simple manual traction when performing TLE, this approach is associated with limited success rates, especially in ICD leads with a dwell time of more than one year. Retrospective studies evaluating the success of different lead extraction techniques at high volume centres report overall success rates of $14 \%$ to $29 \%$ for the simple manual traction approach disregarding the lead type, fixation mechanism, or implant duration [7, 1215]. However, when considering exclusively ICD leads the chances of TLE success with manual traction are lower with around $8 \%$ [13]. The unfavorable lead extraction behaviour of ICD leads can be explained due to a marked fibrous reaction and tissue ingrowth particularly in the interstices between helical turns of the coil wire, anchoring the lead to the venous vasculature or endocardial wall [13]. As a result manufacturers have attempted to mitigate the unwanted effect of tissue ingrowth by producing leads with ICD coils treated with backfilled silicone and medical adhesive or coated with expanded polytetrafluoroethylene (ePTFE). In the subgroup of ePTFE coated ICD leads the extraction success rate is up to $29 \%$ and comparable to non-ICD leads $[13,21]$ and 
adjunct extraction tools were required less frequently with coated leads compared to noncoated leads (39\% versus 63\%) [21]. With regard to this subgroup of ICD leads and to a further trend in implanting single coil, coated ICD-leads simple manual traction will gain importance in removal of defibrillator leads.

\section{Limitations}

Using a simulator always implies certain limitations. Most operators have developed an individual extraction technique modulated by applied traction force or perceived elongation of the lead and also influenced by fluoroscopic feedback and changes in vital signs (e.g., blood pressure and pulse). The latter parameters were not simulated in our model.

Our present model simplifies that the lead is loose in its intravascular or intracardiac route; the next model version will simulate a potential fixation of the outer lead insulation or shocking coil to the vascular or myocardial wall.

In order to standardise our experiment, ICD leads of the same type were used. However, the tensile behaviour of leads may differ between manufacturers and between ICD and pacemaker electrodes. Consequently, the individually acceptable traction force and elongation may vary between different lead models. Thus, further investigations with various leads are warranted.

\section{Conclusion}

A traction force of around $11 \mathrm{~N}$ is considered safe during standard transvenous extraction of ICD leads. The force limit for a safe procedure derived from a pool of experienced extractionists may be helpful for the development of required adequate simulator trainings.

\section{Conflict of Interests}

Carsten Lennerz has received travel support from Sorin, Medtronic, and St. Jude Medical. Herribert Pavaci was supported by a fellowship of the European Heart Rhythm Association (EHRA). Verena Semmler has received travel support from Sorin and St. Jude Medical. Christof Kolb has received lecture fees or travel support from Biotronik, Boston Scientific, Medtronic, St. Jude Medical, and Sorin; he is a consultant to Biotronik and Sorin and performs/has performed clinical studies which were supported by Biotronik, Medtronic, St. Jude Medical, and Sorin.

\section{Acknowledgment}

The authors thank St. Jude Medical Germany for kindly providing the ICD leads for the experiments.

\section{References}

[1] A. J. Greenspon, J. D. Patel, E. Lau et al., "16-Year trends in the infection burden for pacemakers and implantable cardioverterdefibrillators in the United States: 1993 to 2008," Journal of the American College of Cardiology, vol. 58, no. 10, pp. 1001-1006, 2011.

[2] M. K. De Bie, D. A. Fouad, C. J. W. Borleffs et al., "Trans-venous lead removal without the use of extraction sheaths, results of $>250$ removal procedures," Europace, vol. 14, no. 1, pp. 112-116, 2012.

[3] M. P. Brunner, E. M. Cronin, J. Jacob et al. et al., "Transvenous extraction of implantable cardioverter-defibrillator leads under advisory-a comparison of Riata, Sprint Fidelis, and nonrecalled implantable cardioverter-defibrillator leads," Heart Rhythm, vol. 10, no. 10, pp. 1444-1450, 2013.

[4] J. C. Deharo, M. G. Bongiorni, A. Rozkovec et al., "Pathways for training and accreditation for transvenous lead extraction: a European Heart Rhythm Association position paper," Europace, vol. 14, no. 1, pp. 124-134, 2012.

[5] T. Shafat, Y. Baumfeld, V. Novack, Y. Konstantino, and G. Amit, "Significant differences in the expected versus observed longevity of implantable cardioverter defibrillators (ICDs)," Clinical Research in Cardiology, vol. 102, no. 1, pp. 43-49, 2013.

[6] M. G. Bongiorni, C. Blomström-Lundqvist, C. Kennergren et al., "Scientific Initiative Committee, European Heart Rhythm Association. Current practice in transvenous lead extraction: a European Heart Rhythm Association EP Network Survey," Europace, vol. 14, no. 6, pp. 783-786, 2012.

[7] C. Kennergren, C. Bjurman, R. Wiklund, and J. Gäbel, "A single-centre experience of over one thousand lead extractions," Europace, vol. 11, no. 5, pp. 612-617, 2009.

[8] R. G. Hauser, W. T. Katsiyiannis, C. C. Gornick, A. K. Almquist, and L. M. Kallinen, "Deaths and cardiovascular injuries due to device-assisted implantable cardioverter-defibrillator and pacemaker lead extraction," Europace, vol. 12, no. 3, pp. 395-401, 2010.

[9] C. T. Starck, S. P. Salzberg, J. Grünenfelder, T. Kofidis, J. Holzmeister, and V. Falk, "Sondenextraktionen-eine anspruchsvolle Aufgabe," Cardiovascular Medicine, vol. 14, no. 7-8, pp. 213-221, 2011.

[10] B. L. Wilkoff, C. J. Love, C. L. Byrd et al., “Transvenous Lead Extraction: Heart Rhythm Society Expert Consensus on Facilities, Training, Indications, and Patient Management. This document was endorsed by the American Heart Association (AHA)," Heart Rhythm, vol. 6, no. 7, pp. 1085-1104, 2009.

[11] M. Maytin and L. M. Epstein, "The challenges of transvenous lead extraction," Heart, vol. 97, no. 5, pp. 425-434, 2011.

[12] M. G. Bongiorni, E. Soldati, G. Zucchelli et al., "Transvenous removal of pacing and implantable cardiac defibrillating leads using single sheath mechanical dilatation and multiple venous approaches: high success rate and safety in more than 2000 leads," European Heart Journal, vol. 29, no. 23, pp. 2886-2893, 2008.

[13] A. Di Cori, M. G. Bongiorni, G. Zucchelli et al., "Transvenous extraction performance of expanded polytetrafluoroethylene covered ICD leads in comparison to traditional ICD leads in humans," Pacing and Clinical Electrophysiology, vol. 33, no. 11, pp. 1376-1381, 2010.

[14] E. Marijon, S. Boveda, M. De Guillebon et al., "Contributions of advanced techniques to the success and safety of transvenous leads extraction," Pacing and Clinical Electrophysiology, vol. 32, no. 1, pp. S38-S41, 2009.

[15] S. O. Jones IV, R. E. Eckart, C. M. Albert, and L. M. Epstein, "Large, single-center, single-operator experience with transvenous lead extraction: outcomes and changing indications," Heart Rhythm, vol. 5, no. 4, pp. 520-525, 2008. 
[16] C. T. Starck, H. Rodriguez, D. Hürlimann et al., "Transvenous lead extractions: comparison of laser vs. mechanical approach," Europace, vol. 15, no. 11, pp. 1636-1641, 2013.

[17] E. L. Hoover, H. K. Hsu, B. Toporoff, T. Quinn, and I. Thompson, "Use of traction for removing transvenous pacing electrodes in the presence of infection," Journal of the Tennessee Medical Association, vol. 81, no. 8, pp. 504-507, 1988.

[18] A. García-Jiménez, B. C. M. Albá, G. J. M. Cortés, G. C. Rodríguez, A. I. Diéguez, and N. F. Pellejero, "Myocardial rupture after pulling out a tined atrial electrode with continuous traction," Pacing and Clinical Electrophysiology, vol. 15, no. 1, pp. 5-8, 1992.

[19] M. Sohal, S. E. Williams, A. Arujuna et al., "The current practice and perception of cardiac implantable electronic device transvenous lead extraction in the UK," Europace, vol. 15, no. 6, pp. 865-870, 2013.

[20] J. Schläpfer, I. Babotai, U. Bauersfeld et al. et al., "Richtlinien 2011 zur Herzschrittmacher- und Defibrillatortherapie und perkutaner Katheterablation," Cardiocascular Medicine, vol. 14, no. 3, pp. 86-91, 2011.

[21] A. R. Kohut, J. Grammes, C. M. Schulze et al., "Percutaneous extraction of ePTFE-coated ICD leads: a single center comparative experience," Pacing and Clinical Electrophysiology, vol. 36, no. 4, pp. 444-450, 2013. 


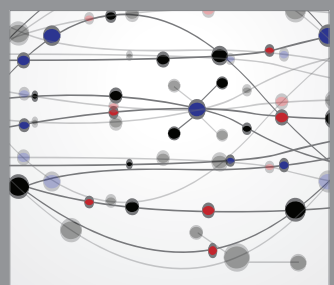

The Scientific World Journal
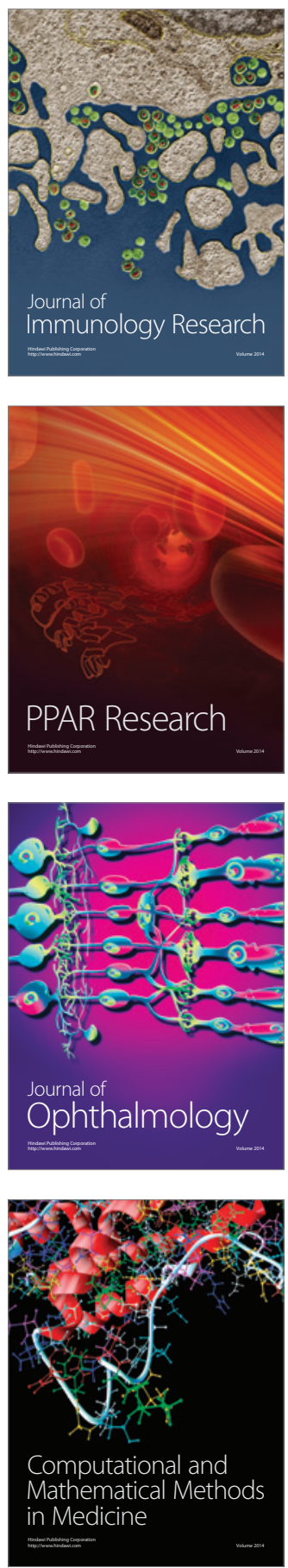

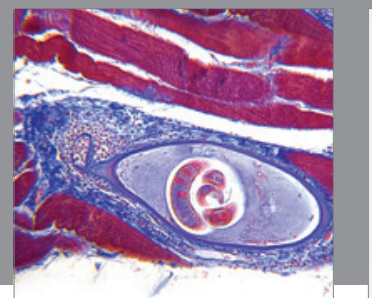

Gastroenterology

Research and Practice
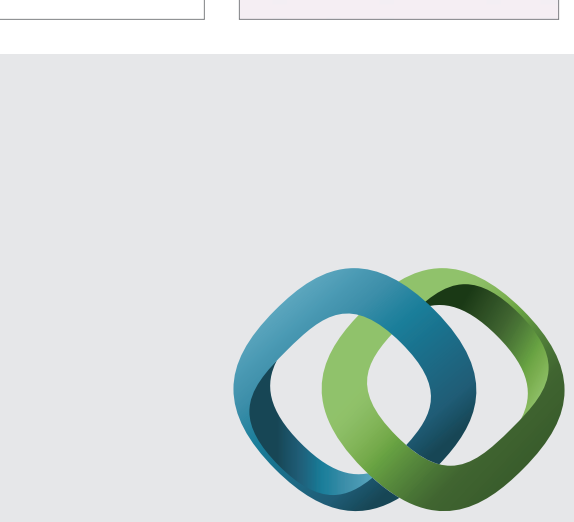

\section{Hindawi}

Submit your manuscripts at

http://www.hindawi.com
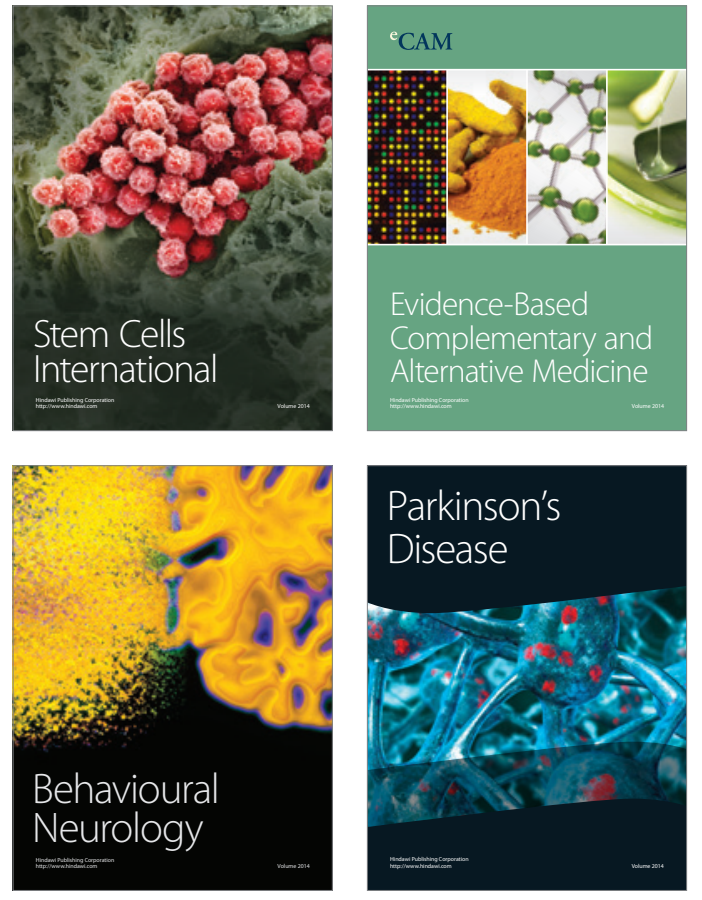
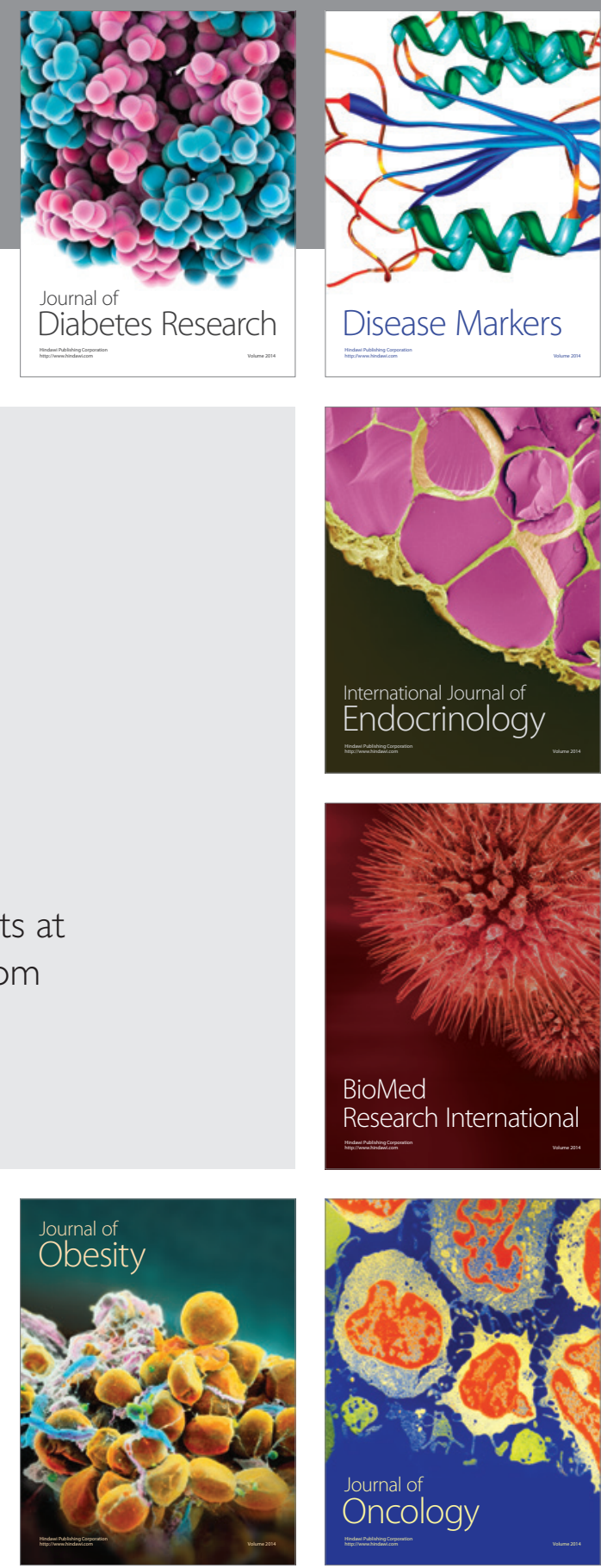

Disease Markers
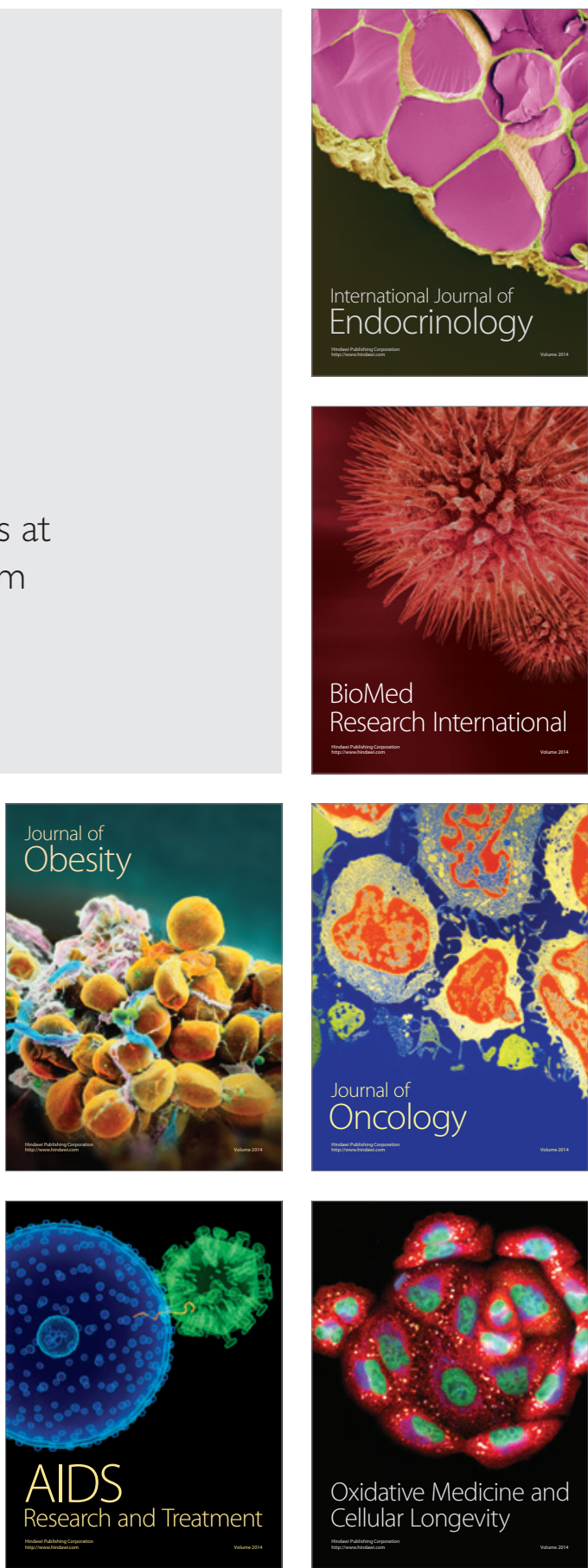